\title{
COMPACTION STRESS IN FINE POWDERS
}

\author{
J.E. SCOTT*, V.M. KENKRE*, E.A. PEASE*, and A. J. HURD** \\ *Department of Physics and Astronomy \\ University of New Mexico, Albuquerque, NM 87131
}

**Sandia National Laboratories, MS 1349, Albuquerque, NM 87185, ajhurd@sandia.gov I I I

\section{ABSTRACT}

APR 20 19ख्ये

A vexing feature in granular materials compaction is density extrema interior to a compacted shape. Such inhomogeneities can lead to weaknesses and loss of dimensional control in ceramic parts, unpredictable dissolution of pharmaceuticals, and undesirable stress concentration in load-bearing soil. As an example, the-centerline density in a. cylindrical compact often does not decrease monotonically from the pressure source but exhibits local maxima and minima. Two lines of thought in the literature predict, respectively, diffusive and wavelike propagation of stress. Here, a general memory function approach has been formulated that unifies these previous treatments as special cases; by analyzing a convenient intermediate case, the telegrapher's equation, one sees that local density maxima arise via semidiffusive stress "waves" reflecting from the die walls and adding constructively at the centerline.

\section{INTRODUCTION}

The fascination with granular materials $[1,2]$ is understandable: When dealing with a thermodynamically significant number of individual, not-too-different agents acting under local rules, sometimes global or cooperative behavior emerges. The grains in a sugar bowl might behave in a way similar to concrete aggregate in a hopper or cereal flakes poured into a bowl. Much of the fascination with physics is in such similarities that cross length scales; but the important task of engineering prediction from a well-grounded, continuum-level model is not always achievable. Judging from the current interest, we may be at a crossroad in understanding powder compaction.

Considerable activity is apparent in the recent literature $[1,2,3,4,5,6,7,8,9,10$, $11,12,13,14,15]$ in the calculation of the stress distribution in granular materials ranging from sandpiles to ceramic powder compacts. The interest stems in part from new experimental results $[16,17,18]$, in part from the fact that important features of old experimental data $[19,20,21]$ have never been satisfactorily explained, and in part from the need to understand and control the formation of undesirable density gradients in the manufacture of ceramic parts. The behavior of granular materials under compressive stresses is poorly understood, despite the 140 years of research since Rankine investigated stress distributions in loose soil [22]. Studies in this field continue with a high level of intensity: in civil and geological engineering under the topic of soil mechanics; in mechanical, ceramic, and metallurgical engineering in the context of shrinking, cracking and failure during the pressing and sintering processes; and in general theoretical physics in the context of self-organized criticality with implications toward the understanding, of geodynamic processes such as earthquakes and avalanches.

The goal of this work, recently accepted for publication in Physical Review E [11], is to find the weakest assumptions connecting existing theoretical treatments that cover successfully accepted measurements. That connection is made here through a constitutive relation between stress tensor components, generalized only enough to envelope two atfirst-sight unrelated theories. Expressed as a memory function, the "connector" provides a fascinating spectrum of prediction that reproduces known theory at its limits but offers new insights between. 


\section{DISCLAIMER}

Portions of this document may be illegible in electronic image products. Images are produced from the best available original document. 


\section{THE COMPACTION PROBLEM}

The "hydrogen atom" of powder compaction is a right circular cylinder or "die," closed at one end and filled with loose grains that are compacted from the open end. Many experiments have been done in this geometry and a few incontrovertible facts have been established, reviewed below. Although the geometry may seem somewhat special from the standpoint of applications-however surprisingly many products from ceramics to pills have this basic shape-it has enough richness to be theoretically challenging. Generally, two approaches have been used to obtain experimental information about the propagation of stress in a powder compact: 1) direct measurement of the stress at points within a compact, and 2) direct mapping of the density distribution or displacements within a compact. The first approach involves the use of pressure sensors or strain gauges within, or at the edge of, a compact to measure the forces that evolve during pressing. Such measurements are limited by their accuracy and the ability of such gauges to measure the direction and location of the transmitted stress. The second approach relies on the assumption that the density at a point in a compact is related to the magnitude of the transmitted applied stress at that point through an "equation of state." Various equations of state exist to describe density-pressure behavior for granular systems [12-15].

Stress distribution observations include the experiments of Duwez and Zwell [16] who measured the pressure at the side wall of a cylindrical compact of copper powder as a function of depth. The side pressure was found to decrease linearly with distance away from the surface where the pressure was applied_ Irain [9] placed strain gauges within a fill of magnesium carbonate powder in a cylindrical die and measured the distribution of transmitted stress as a function of applied pressure during the compaction process. Along the centerline of the compact, the transmitted stress was-observed to be a non monotonic function of the depth.

The measurement of density distributions are exemplified by the work of Kamm, Steinberg and Wulff [7] who placed a thin lead grid into a cylindrical copper powder fill which was subsequently pressed. The distortion of the grid was observed using X-rays to determine the local displacements hence density distribution of the compact. High and low density regions were observed along the centerline of the compact, while the density at the edge of the compact decreased from high density in the upper comers to low density at the bottom. Kuczynski and Zaplatynsky [8] determined the density distribution of a nickel powder compact by sectioning it and testing the hardness of the compacted material as a function of location on the face of the section. By assuming a relation between the density of the material to its hardness, a density distribution was produced showing behavior similar to that seen by Kamm, etal_ [7]. The density along the top surface of the compact had a minimum in the center and increased to a maximum value at the edge of the compact.

Train [9] measured the density distribution of the magnesium carbonate compacts that he used in his direct stress measurements by machining away sections and measuring the weight and volume of the material removed. Contour plots of the density distribution were 


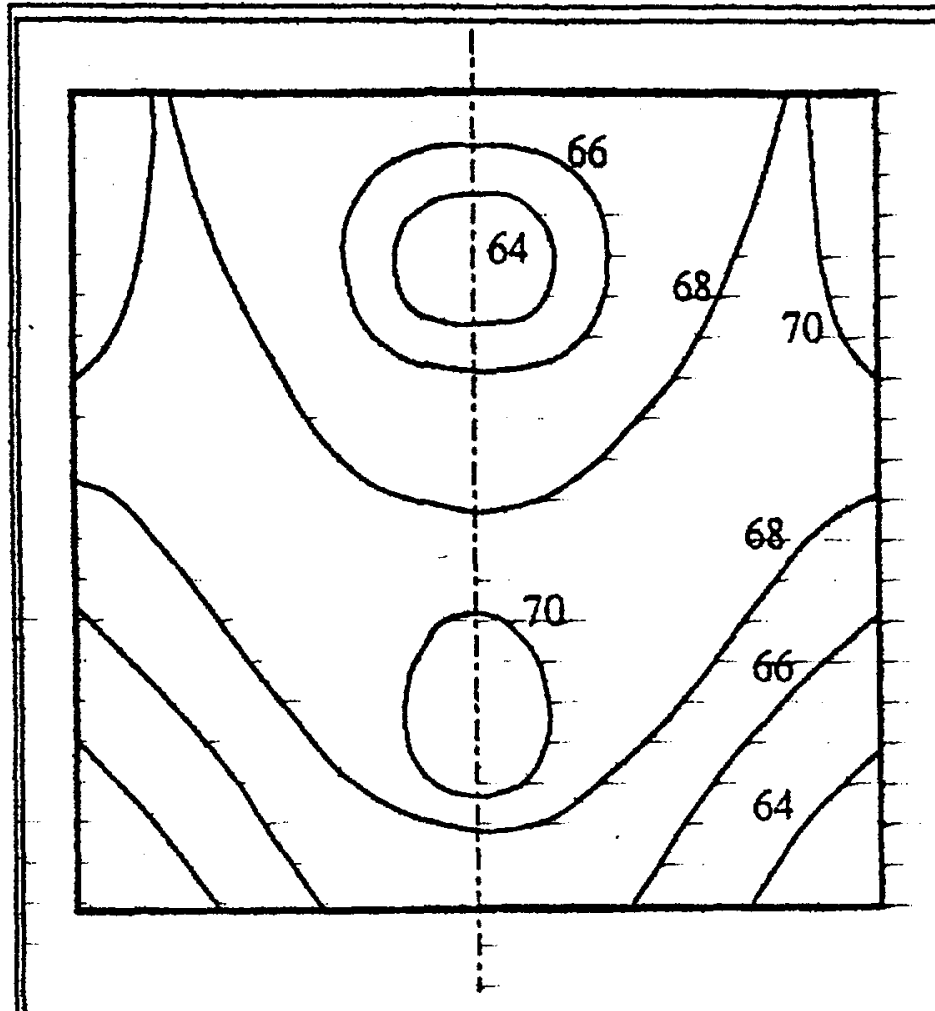

Figure 2 Density distribution in percent solid density for cylindrical compacts of magnesium carbonate powder pressed at 200 MPa showing interior density extrema (from Train [9]).

produced showing the density for several different applied pressures. Well defined regions of high and low density along the centerline were observed, as well as the decreasing density along the edge of the compact.

Macleod and Marshall [10] compacted uranium dioxide powder in a cylindrical die for various applied pressures, then - sectioned the compacts and placed them on photographic plates. - Density distributions were obtained using contact autoradiography, where the exposure was calibrated by comparison with the optical density of plates that were - exposed to uranium dioxide pellets with known densities. The contour plots they published - showed non-monotonic-behavior - alo the centerline of the - compacts, with several regiens of fhigh and low density. Along the top-surface, the density as a F function of radius, increasing from a minimum at the center to a maximum at the edge of the compact, similar to the observations of Kuczynski and Zaplatynsky [8].

Recently, Aydin, Briscoe and Sanliturk $[6]$ imbedded small lead balls in an alumina powder compact and used a technique similar to $\mathrm{Kamm}$, et al. [7] to determine the density distributions. Regions of high and low density were observed on the centerline, with the density at the edge of the compact decreasing with depth. Along the top surface of the compact, the density distribution, as a function of the radius, exhibited a minimum at the centerline and increased toward the edge of the compact, consistent with previous observations [8,10].

- Three features describing the stress distribution in cylindrical compacts can be extracted:

1) the applied stress at the top-surface is notconstant, but increases from a-minimum at the center to a maximum at the edges,

2) the stress at the edges decreases with depth from the surface where the pressure is applied, and

3) the stress along the centerline is a non monotonic function of depth, with yell defined regions of low and high stress.

These features, which are common to observations made in a variety of powders over a wide range of applied pressures, provide inputs as well as a test for theoretical treatments of the stress distribution in compacts: no theoretical analysis known to us has adequately addressed the appearance of non-monotonic stress behavior along the axis. 


\section{EXTANTTHEORIES}

Ianssen [17] developed in 1895 a model to describe the pressure distribution along grain silo walls, showing an exponential decrease in pressure along the walls as a function of depth. Thompson [13] extended this work to the compaction of ceramic powders by assuming that the shear stress at the die wall was due to dynamic friction only, that the dynamic friction followed the Coulomb friction condition, and that the friction wall force was directly proportional to the radial stress normal to the wall. The resulting model reproduced the exponentially decreasing stress along the die wall, but predicted constant stress along the centerline of the compact in disagreement with observations. Both authors used the Cauchy relation,

$$
\nabla \cdot \sigma+\rho b=\rho \dot{v}
$$

to develop a continuum theory. Here $\sigma$ is the stress tensor, $\rho b$ is all body forces such as gravity, and the $z$ axis runs parallel to the cylinder. In the quasistatic limit, they assumed the proportionality of $\sigma_{\mathrm{xx}}, \sigma_{\mathrm{yy}}$, and $\sigma_{\mathrm{z}}$ as well as the vanishing of shear in the $\mathrm{x}-\mathrm{y}$ plane, $\sigma_{x y}=\sigma_{y x}=0$. These additional relations are constitutive or closure relations about which all the controversy around continuum theories spins.

Edwards and collaborators $[3,4]$ developed a statistical mechanical approach that described the transmission of stress away from the center of the compact to the walls through "arches" of granular particles that were aligned in such a way as to support the compact using wall friction Stress equations and constitutive relations were postulated and applied to bounded systems, but the distribution of stress in compacts was not calculated.

Our purpose in the present paper is to provide a theoretical framework based on the formal interpretation of the vertical spatial coordinate as time, described next. Such an idea appears in at least two earlier analyses in the literature One of them is by Bouchaud, et al. [5] who use continuum mechanics to derive a "wave equation" to describe the "coherent" transmission of stress in a granular compact. The other, although it might not bave heen recognized as such, is the quite unrelated analysis of Liu, et al. [18] who describe stress distribution through the use of a discrete master equation following what may be regarded as a Markoffian evolution.

\section{THE Z TRANSFORMATION}

Because interest lies primarily in the local density in the compact, which is believed to be a function of the local value of $\sigma_{z z}$, we will focus first on the $z$ component of $E q$. (1) which governs this quantity It can be recast in the form of a 2-dimensional continuity equation

$$
\frac{\partial \sigma_{x}}{\partial x}+\frac{\partial \sigma_{x}}{\partial y}+\frac{\partial \sigma_{z z}}{\partial z}=-p g \Rightarrow \nabla \cdot j+\frac{\partial S}{\partial z}=-p g
$$

The "flux" $\mathrm{j}$ is a two-dimensional vector with $\sigma_{\mathrm{xz}}$ and $\sigma_{\mathrm{rz}}$ as its $\mathrm{x}$ and y components respectively, the "density" $S$ of the fictitious fruid whose flow is given by equation (2) is identical to $\sigma_{z}$, and $\rho g$ is a "source-term" representing downward gravity If the applied stresses are much larger than the gravitational force, the source term can be neglected. In this interpretation, the $\mathrm{z}$ coordinate assumes the role of time, by analogy to common transport phenomena, 


$$
\frac{\partial S}{\partial t}=-\nabla \cdot \mathbf{j}+\text { sources } .
$$

Equation (3), which we now consider with its sources put equal to zero, can be used to determine $S$ only if an additional equation relating the components of $j$ to $S$ is given. In order to understand the spirit of our analysis below, it is useful to consider three examples from fluid flow of such an additional constitutive equation. The first example is Fick's law,

$$
\mathrm{j}=-D \nabla S \text {, }
$$

which, when substituted in the continuity equation, leads to the diffusion equation for $S$ with $D$ as the diffusion constant The symbol $\nabla$ here represents the two dimensional gradient.

The second example is

$$
\frac{\mathrm{g}}{\partial z}=-c^{2} \nabla S,
$$

and describes the proportionality between the "time" derivative ( $z$ derivative) of the flux and the gradient of the density. It leads to the wave equation, with wave propagation speed $\mathrm{c}$. The third example combines features of the first and the second examples,

$$
\frac{\partial}{\partial z}+\frac{c^{2}}{D} \mathbf{j}=-c^{2} \nabla S,
$$

and reduces to the previous two limits in easily understood extreme limits. When substituted in the continuity equation, (6) leads to what is known as the telegrapher's equation [12,20]

$$
\frac{\partial^{2} S}{\partial z^{2}}+\frac{c^{2}}{D} \frac{\partial S}{\partial z}=c^{2} \nabla^{2} S
$$

used to determine the optimum spacing between telegraph poles. This telegrapher's. equation, which we will find of considerable use in the present paper, unifies diffusive and wave behaviors in a straightforward fashion and is itself a special case of a generalized memory equation [21] where the constitutive relation is non-local in the $\mathrm{z}$ coordinate

$$
\mathbf{j}(z)=-D \int_{0}^{z} d z^{\prime} \phi\left(z-z^{\prime}\right) \nabla S\left(z^{\prime}\right)
$$

The resulting equation for $S$,

$$
\frac{\partial S(z)}{\partial z}=D \int_{0}^{e} d z^{\prime} \phi\left(z-z^{\prime}\right) \nabla^{2} S\left(z^{\prime}\right),
$$

reduces to the diffusion, the wave, and the telegrapher's equations in the respective-limits of a "memory" that vanishes $\phi(z)=\delta(z)$, is constant $\phi(z)=c^{2} / D$, and is intermediate $\phi(\mathrm{z})=\left(\mathrm{c}^{2} / \mathrm{D}\right) \exp \left[-\left(\mathrm{c}^{2} / \mathrm{D}\right) \mathrm{z}\right]$ ! 
We will see below that an examination of the common constitutive relations assumed in the literature suggests a natural generalization. That generalization leads to the nonMarkoffian evolution Equ (9) reducing in a simple instance to the telegrapher's Equ. (7), and it is possible on the basis of these equations to construct a detailed framework for the description of stress distribution in granularmedia.

\section{WEAK ASSUMPTIONS FOR STRONG RELATIONS}

In the present state of the theory of stress distribution in granular materials, constitutive relations form the weakest link because, whether made explicit or implicit in the analysis, they are ad hoc in nature Nevertheless, such relations are judged pragmatically by whether they solve engineering problems.

This situation is not the result of lack of effort. It appears extremely difficultat the present stage of the field to provide any satisfactory physical justification for existing relations As in the case of other consitutive relations in the literature, the one we propose is also ad hoc. However we will see that in a certain sense it is mathematically natural. Furthermore, it has the distinctadvantage of leading to a unification and generalization of earlier treatments.

The closure assumption of Janssen [17] and Thompson [13] particularly as expressed by Bouchaud, et al. [5] postulates proportionality between the diagonal elements of the : stress tensor as well as the vanishing of the shear components in the $x-y$ plane-In the second part of their analysis, Bouchaud, et al. attempt to go beyond this assumption through the inclusion of nonlinear, second order corrections in $\sigma_{x z}$ and $\sigma_{y z}$. Following Thompson's lead, in the absence of a satisfactory physical argument the most natural constitutive relation is a simple proportionality

$$
\sigma_{x x}=\text { const } \cdot \sigma_{z z} ; \sigma_{y y}=\text { const } \cdot \sigma_{z z} \text {. }
$$

Wenote, however, that this relation is never used directly for combining with (1) butonly in the form of spatial derivatives,

$$
\frac{\partial \sigma_{x x}}{\partial x}=c_{1}^{2} \frac{\partial \sigma_{z y}}{\partial x} ; \frac{\partial \sigma_{y y}}{\partial y}=c_{2}^{2} \frac{\partial \sigma_{z}}{\partial y},
$$

where $c_{1}$ and $c_{2}$ are equal constants in isotropic media. One expects additional terms representing shear (not in the $x$-y planel) coupling between the diagonal terms, and in the spirit of a Taylor expansion only first-order terms are adopted,

$$
\frac{\partial \sigma_{x x}}{\partial x}=c_{1}^{2} \frac{\partial \sigma_{z}}{\partial x}+\alpha_{1} \sigma_{x z} ; \frac{\partial \sigma_{y y}}{\partial y}=c_{2}^{2} \frac{\partial \sigma_{z}}{\partial y}+\alpha_{2} \sigma_{x},
$$

and again $\alpha_{1}$ would equal $\alpha_{2}$ in an isotropic medium. Combining with Eq. (1) and assuming that transverse shear vanishes $\sigma_{x y}=\sigma_{y x}=0$, we obtain

$$
\frac{\partial}{\partial z}+\alpha_{1} j_{x} \hat{x}+\alpha_{2} j_{y} \hat{y}=-\left(c_{1}^{2} \frac{\partial S}{\partial x} \hat{x}+c_{2}^{2} \frac{\partial S}{\partial y} \hat{y}\right) \text {. }
$$

Equation (13) or its isotropic counterpart (which we will use in most of the analysis below) is our new constitutive relation It leads to the telegrapher's equation (7) for $S$. Whilewe have assumed above, following $R e f$. [5], that $\sigma_{x y}$ and $\sigma_{y x}$ are identically equal to zero, it is 
actually not necessary to make this assumption to get (13): It is-enough to postulate-that (12) is replaced by

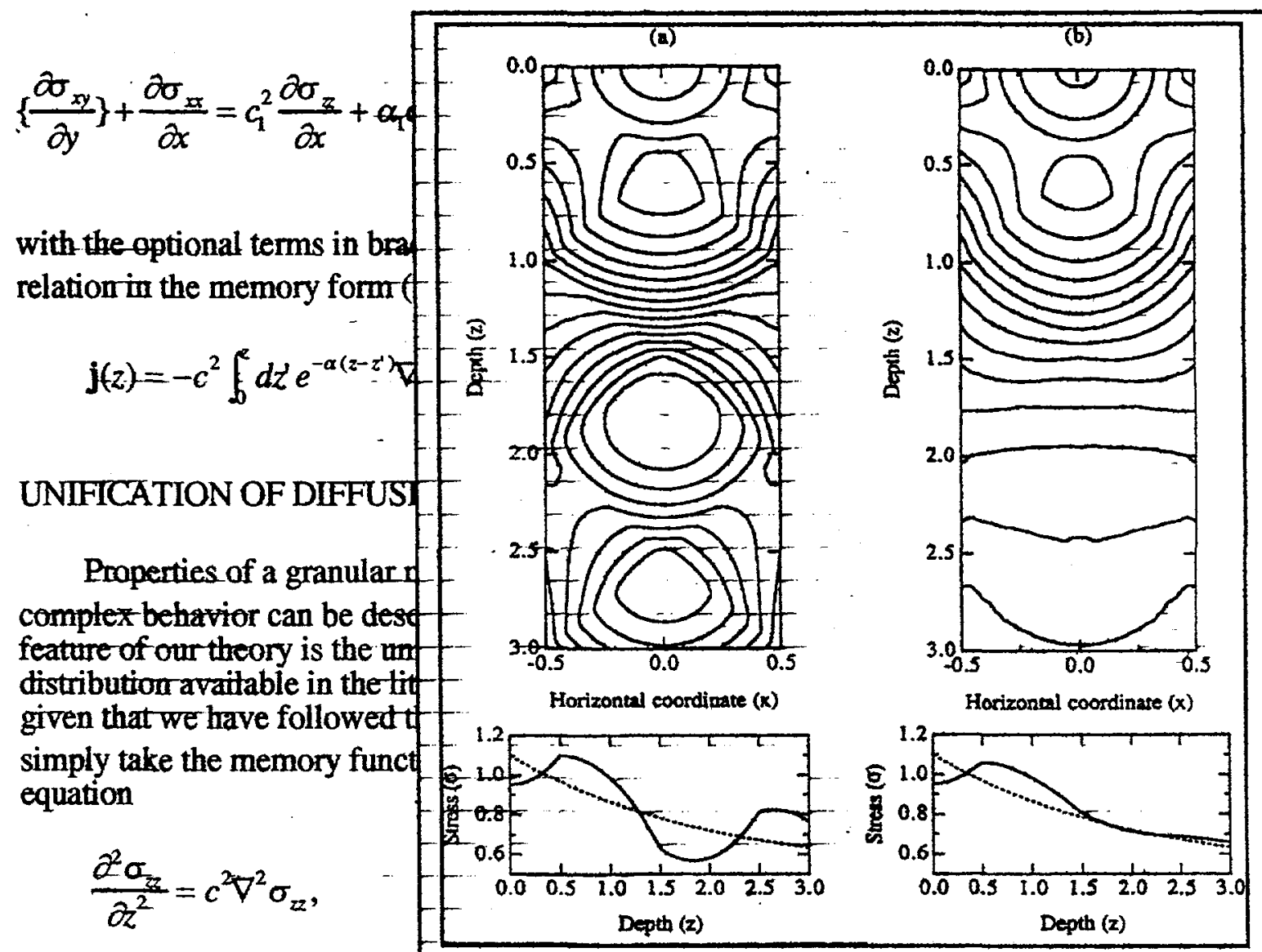

which; with the identification interpretation here is that the [5] describes the perfect reten layer in the $z$ direction. Such frictionless spherical particies one particle would be transmi would be no loss of informati force.

This coherent limit does randomly shaped particles of Randomness would suggest t Markoffian processes charact

Figs. 3a-d. Calculated stress distributions using equation (7) showing the transition from fully wave-like stress propagation to diffusive propagation. The plots below the contour plots show the centerline stress compared to the exponential boundary condition. (a) $c=1.0$, $\alpha=0.0$, (b) $c=1.0, \alpha=2.0$, (c) $c=1.3$. $r=10.4$. and
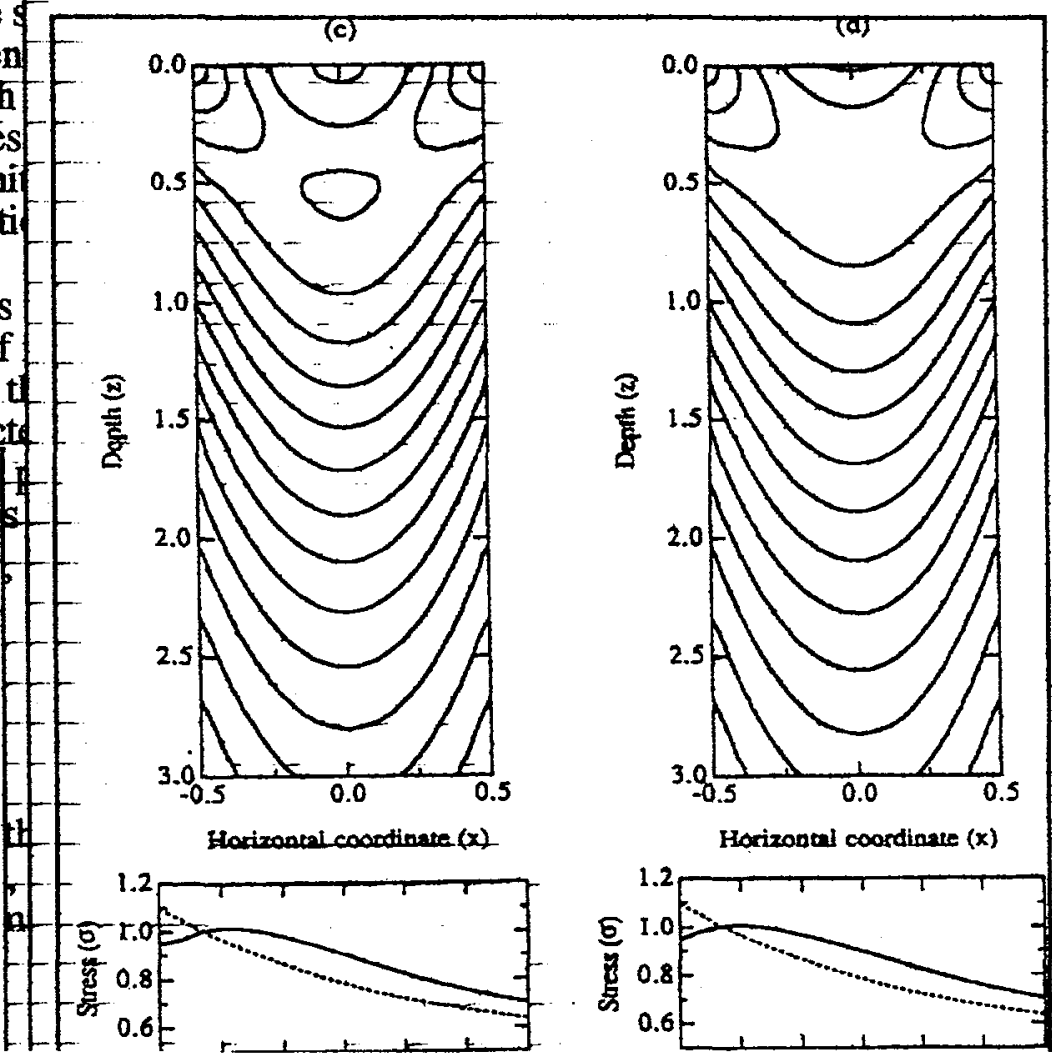
[7] R Kamm,M. A. Steinberg, and I Wulff, Trans AIME 171, 432(1947); 180, 694 (1949).

[8] G. C. Kuczynski and I Zaplatynsky, Trans, AIME 206, 215 (1956).

[9] D. Train, Trans. Instn. Chem. Engrs. 35, 258 (1957).

[10]-H. Macleod andK_Marshall,Powder Technology 16,-107 (1977).

[11] V. M. Kenkre, J. E. Scott, E. A. Pease, and A. J. Hurd, Phys. Rev. E, (parts I and II, in press, 1997).

[12] R. P. Seelig, Trans. AIME 171, 506 (1947).

[13] R_A Thompson, Ceramic Bulletin 60, 237 (1981)

[14] A. R. Cooper and L. E. Eaton, J. Am. Ceram. Soc. 45, 97 (1962).

[15] V_M Kenkre, M L R Endicatt,S I Glass, and A I Hurd, J. Am Ceram. Soc. 79, 3045 (1996)

[16]P. Duwez-and L. Zwell, Trans-AIME 185, 137 (1949).

[17] H. A. Janssen, Z. Ver. Dt. Ing. 39, 1045 (1895).

[18] C, -h Liu, S. R. Nagel, D A Schecter, S N Coppersmith, S Majumdar, O. Narayan, T. A. Witten, Science 269, 513 (1995).

[19] V. M Kenkre, in Energy Tranefer Processes in Condensed_Matter edited by B Di Bartolo (Plenum Press, New York, 1984), pp. 205-249.

[20] P M. Morse and H_. Feshbach, Methods of Theoretical Physics, Volume 1,
McGraw Hill Book Company, Inc., New York, 1953, p. 865 . [21] K. M. Kenkre and P. Reineker, Exciton Dynamics in Molecular Crstals and Aggregates. Springer-Verlag, New York, 1982.

[22] S. I Farlow, Partial Differential Equations for Scientists and Engineers_Daver Publications, Inc., New York,.1982, pp. 64-73. 